\title{
Spin resonance amplitude and frequency of a single atom on a surface in a vector magnetic field
}

\author{
Jinkyung Kim,,${ }^{1,2,}{ }^{*}$ Won-jun Jang, ${ }^{3,},{ }^{*}$ Thi Hong Bui, ${ }^{1,2}$ Deung-Jang Choi $\odot,{ }^{4,5,6}$ Christoph Wolf $\odot,{ }^{1,7}$ Fernando Delgado $\odot,{ }^{8}$ \\ Yi Chen, ${ }^{1,7}$ Denis Krylov, ${ }^{1,7}$ Soonhyeong Lee, ${ }^{1,7}$ Sangwon Yoon, ${ }^{1,7}$ Christopher P. Lutz $\bullet,{ }^{9}$ \\ Andreas J. Heinrich, ${ }^{1,2, \dagger}$ and Yujeong Bae ${ }^{1,2, 末}$ \\ ${ }^{1}$ Center for Quantum Nanoscience, Institute for Basic Science, Seoul 03760, South Korea \\ ${ }^{2}$ Department of Physics, Ewha Womans University, Seoul 03760, South Korea \\ ${ }^{3}$ Samsung Advanced Institute of Technology, Suwon 13595, South Korea \\ ${ }^{4}$ Centro de Física de Materiales-MPC (CSIC-UPV/EHU), 20018 San Sebastián, Spain \\ ${ }^{5}$ Donostia International Physics Center, 20018 Donostia, San Sebastian, Spain \\ ${ }^{6}$ Ikerbasque, Basque Foundation for Science, 48013 Bilbao, Spain \\ ${ }^{7}$ Ewha Womans University, Seoul 03760, Republic of Korea \\ ${ }^{8}$ Instituto de Estudios Avanzados IUDEA, Departamento de Fisica, Universidad de La Laguna, 38203 Tenerife, Spain \\ ${ }^{9}$ IBM Almaden Research Center, San Jose, California 95120, USA
}

(Received 30 March 2021; revised 25 August 2021; accepted 30 September 2021; published 8 November 2021)

We investigated spin-1/2 hydrogenated titanium (Ti) atoms on $\mathrm{MgO}$ using scanning tunneling microscopy (STM) combined with electron spin resonance (ESR) in vector magnetic fields. Rotating external magnetic fields, we observed rather drastic changes in both amplitude and frequency of resonance signals for single Ti atoms. While the variation of ESR amplitudes reflects the effects of the spin polarization of a magnetic tip and local magnetic fields created by the interaction between the tip and $\mathrm{Ti}$, the change of resonance frequencies shows the anisotropy of $g$ values for Ti atoms. Using the Ti atoms at the low-symmetry bridge adsorption site of the $\mathrm{MgO}$ lattice allowed for identifying the $g$ values in all three spatial directions. Multiplet calculations confirmed the origin of this anisotropy as the spin-orbit coupling induced effects of crystal. Our results show the capability of single atomic spins as a sensor to probe magnetic surroundings and highlight the precision of ESR-STM to identify the single atom's spin states in a solid-state environment.

DOI: 10.1103/PhysRevB.104.174408

\section{INTRODUCTION}

Single spins are widely regarded as an excellent candidate for realizing next-generation quantum devices for sensing [1-3] and information processing [4]. When placed in a solidstate environment, single spin centers, such as adatoms or point defects in a crystal, can be strongly influenced by their local environments of neighboring electron and nuclear spins [5,6], orbital and valley angular momenta [7-9], and static and fluctuating magnetic fields [10]. It is thus important to develop a technique that allows us to characterize the interactions between a spin center and its immediate surroundings.

Electron spin resonance (ESR) offers high energy resolution for the measurement of spins, which is not limited by the temperature. Scanning tunneling microscopy (STM), on the other hand, offers access to individual spins and to the surrounding environment at the atomic scale, albeit with an energy resolution that is limited by the temperature of tip and sample [11-13]. Recently, it was shown that these advantages could be combined in ESR-STM, which achieves an energy resolution of tens of neV with atomic-scale spatial resolution on individual spins $[14,15]$.

\footnotetext{
${ }^{*}$ These authors contributed equally to this work.

${ }^{\dagger}$ Corresponding author: heinrich.andreas@qns.science

${ }^{\ddagger}$ Corresponding author: bae.yujeong@qns.science
}

In this paper, we positioned and probed a hydrogenated titanium (Ti) atom with spin 1/2 on $\mathrm{MgO} / \mathrm{Ag}(100)$ using ESRSTM incorporated with a vector magnet. In such a setup, the atomic spin in the tunnel junction is subjected to a magnetic field provided by not only an external magnetic field but a spatially inhomogeneous magnetic field of tip. Two strengths of $\mathrm{Ti}$ on $\mathrm{MgO}$ make it desirable for study to identify the surface atom's magnetic surroundings which influence its spin state and thus its spin resonance. First, no zero-field splitting exists for spin $1 / 2$ [16], and so the Ti spin follows the twodimensional external magnetic field direction and acts as a rotatable sensor in the $x z$ plane [Fig. 1(a)]. Second, the sizeable spin-orbit coupling of Ti makes it a sensitive probe to the substrate crystal field, the influence of which can be quantified by characterizing the $g$ factor of Ti along the three principal axes. By rotating the single spin using the static external magnetic field, we are able to obtain a full spatial characterization of the tip magnetic field and the three-dimensional map of the surface spin's $g$ factor from extensive ESR spectroscopic measurements.

\section{METHOD}

\section{A. Experimental setup of single atom ESR measurement in low-temperature STM}

We performed ESR measurements of single atoms on an ultraclean $\mathrm{MgO}$ surface using a home-built ultra-high-vacuum 

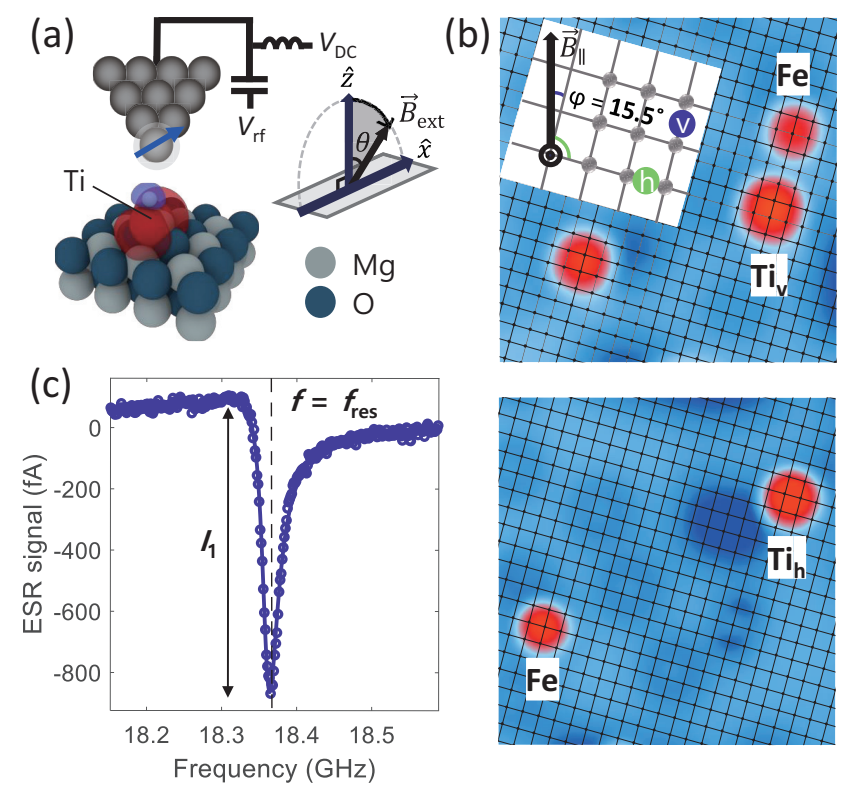

FIG. 1. The hydrogenated $\mathrm{Ti}$ atom on a bridge binding site $\left(\mathrm{Ti}_{\mathrm{B}}\right)$ of $\mathrm{MgO}$ and corresponding ESR spectrum in a vector magnetic field. (a) Schematic of the ESR-STM setup combined with the spin density of $\mathrm{Ti}_{\mathrm{B}}$ on $\mathrm{MgO}$ calculated by DFT. The external magnetic field rotates in a two-dimensional plane ( $x z$ plane) with the angle $\theta$, which is measured relative to the sample surface normal ( $z$ direction). (b) STM images of $\mathrm{Ti}_{\mathrm{B}}$ atoms with different binding configurations on $\mathrm{MgO}\left(V_{\mathrm{DC}}=100 \mathrm{mV}, I_{\text {set }}=20 \mathrm{pA}\right.$ (top) and $10 \mathrm{pA}$ (bottom), and $T=1.12 \mathrm{~K}$ ). The intercepts of grid lines represent the positions of oxygen atoms of the $\mathrm{MgO}$ lattice and were aligned using $\mathrm{Ti}$ and $\mathrm{Fe}$ atoms on top of oxygen as markers. Inset: The angle between the in-plane direction of the external magnetic field $\left(B_{\|}\right)$and a line connecting two neighboring oxygen atoms is $\varphi$. When $\varphi=15.5^{\circ}$, $\mathrm{Ti}_{\mathrm{B}}$ atoms are labeled as $\mathrm{Ti}_{\mathrm{v}}$. In the other case $\left(\varphi=105.5^{\circ}\right)$, they are labeled as $\mathrm{Ti}_{\mathrm{h}}$. Note that $\varphi=105.5^{\circ}\left(\mathrm{Ti}_{\mathrm{h}}\right)$ is equivalent to $\varphi=74.5^{\circ}$ due to the twofold symmetry. (c) ESR spectrum of $\mathrm{Ti}_{\mathrm{v}}$ measured at $B_{\text {ext }}=0.7 \mathrm{~T}$ and $\theta=90^{\circ}$ using tip $1\left(V_{\mathrm{DC}}=40 \mathrm{mV}, I_{\text {set }}=20 \mathrm{pA}\right.$, $V_{\mathrm{RF}}=10 \mathrm{mV}$, and $T=1.12 \mathrm{~K}$ ), and the solid line is the best fit to Eq. (1).

(UHV) scanning tunneling microscope with high-frequency transmission cables. The system can reach a base temperature of $30 \mathrm{mK}$ using a dilution refrigerator system (Janis Research, JDR-250) when operated with ${ }^{3} \mathrm{He}-{ }^{4} \mathrm{He}$ gas mixture. Throughout this paper, we measured ESR spectra at $1.12 \mathrm{~K}$ using only a small amount of mixture gas in the Joule-Thomson effect. The system is equipped with superconducting magnets which provide a vector magnetic field: maximum $9 \mathrm{~T}$ in the in-plane direction, $4 \mathrm{~T}$ in the out-of-plane direction, and $3 \mathrm{~T}$ when two magnets are simultaneously used.

Iron $(\mathrm{Fe})$ and $\mathrm{Ti}$ atoms were subsequently deposited on two monolayers of $\mathrm{MgO}$ on $\mathrm{Ag}(100)$ at a temperature lower than $15 \mathrm{~K}$. All adatoms on $\mathrm{MgO}$ can be easily identified by their apparent height in the STM images as well as by their unique spectral features in scanning tunneling spectroscopy (see Sec. S2 in the Supplemental Material [17]). As previously observed, Ti atoms exist in a hydrogenated state after absorbing residual hydrogen from the UHV chamber $[16,18]$. For simplicity we will refer to these hydrogenated $\mathrm{Ti}$ atoms as $\mathrm{Ti}$ atoms from here on. The Ti atoms were found at two different binding sites: one on top of oxygen of the $\mathrm{MgO}$ lattice and the other at the bridge site between two oxygen atoms. In this paper, we measured ESR on the Ti atoms at the bridge binding sites $\left(\mathrm{Ti}_{\mathrm{B}}\right)$ and used three different tips for the ESR measurements.

\section{B. Calculations of the $g$-factor anisotropy}

We performed magnetic multiplet calculations based on density functional theory (DFT) as previously reported in Ref. [19]. DFT calculations using pseudopotentials and plane-wave bases were performed in QUANTUM ESPRESSO (version 6.5) [20] with Perdew-Burke-Ernzerhof pseudopotentials from the standard solid-state pseudopotentials table (precision) [21] with a kinetic cutoff for the plane waves of $60 \mathrm{Ry}$ and a dual of 10. The Brillouin-zone integration was performed on a $3 \times 3 \times 1$ Monkhorst-Pack $k$ grid and dispersive forces were treated using Grimme's van der Waals correction [22].

Following previous reports, we modeled our system as two monolayers of $\mathrm{MgO}$ frozen at the lateral lattice constant of silver $\left(a_{\mathrm{Ag}, \mathrm{DFT}}=4.16 \AA\right)$, expanded into a $1 \times 1 \mathrm{~nm}^{2}$ lateral supercell and padded by $15 \AA$ of vacuum in the $z$ direction [23]. A Ti atom was added to the cell above a bridge site between two oxygen atoms of $\mathrm{MgO}$ and the system was relaxed until all forces were less than $10^{-3}\left(\mathrm{Ry} / a_{0}\right)$. Then a hydrogen atom was added and the whole system was relaxed again. The relaxed structure was used to extract the coordinates and Lowdin charges were used as point charges in the multiplet calculations to represent the crystal field $[24,25]$. To accurately treat the Ti $3 d$ manifold we are using scaled hydrogenic wave functions for spin-orbit coupling and electron-electron terms. The spin-orbit coupling strength was scaled to $90 \%$ of its value obtained from gas phase calculations $(\zeta=18.948 \mathrm{meV}$ ) [26], necessarily reduced due to the screening from the $\mathrm{MgO}$ substrate. The $3 d-3 d$ electron repulsion was scaled to $6.3 \mathrm{eV}$ and the crystal field was strongly screened by $75 \%$. The strong screening of the crystal field is most likely a consequence of significant on-site $3 d-4 s$ hybridization. Indeed, we find that the DFT wave function corresponding to the singly occupied $d_{x^{2}-y^{2}}$ state of Ti has about $10 \%$ contribution from the $4 s$ orbital (see Sec. S5 in the Supplemental Material [17]).

\section{RESULTS AND DISCUSSION}

\section{A. ESR spectra on Ti atoms with twofold symmetry}

$\mathrm{Ti}$ atoms adsorbed at a bridge binding site of $\mathrm{MgO}$ can be seen by STM topography [Fig. 1(b)] and show two inequivalent binding sites with twofold symmetry. Their position can be easily changed by atom manipulation. The difference of two bridge binding sites originates from the relative angle of the $\mathrm{MgO}$ lattice with the external magnetic field and thus the $\mathrm{Ti}$ spin [27,28]. We label $\mathrm{Ti}$ at the two different bridge binding sites as vertical $\mathrm{Ti}_{\mathrm{v}}$ and horizontal $\mathrm{Ti}_{\mathrm{h}}$. For $\mathrm{Ti}_{\mathrm{v}}$, the nearest neighbor oxygen atoms were almost aligned with the in-plane component of the external magnetic field, while they were almost perpendicular in the case of $\mathrm{Ti}_{\mathrm{h}}$ [Fig. 1(b), inset]. By using these two inequivalent sites, we can determine the 

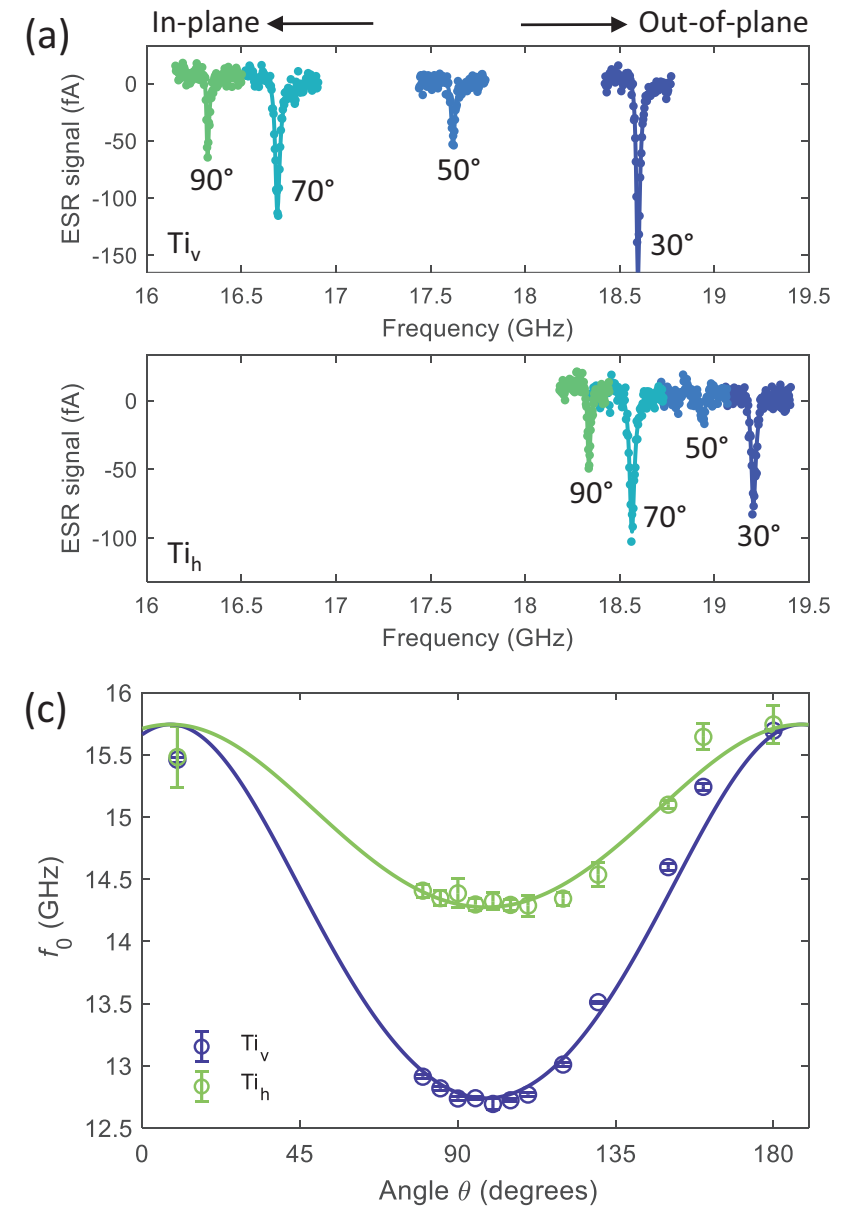
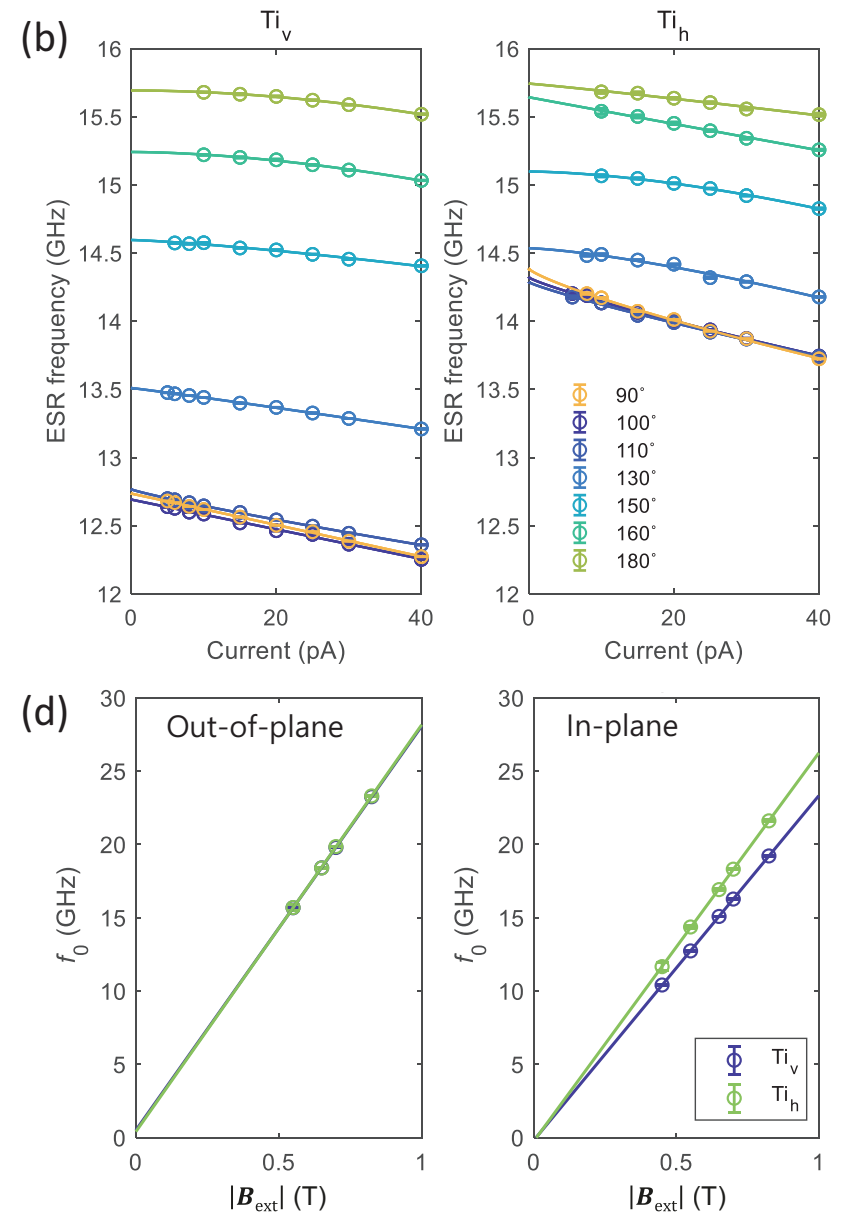

FIG. 2. Anisotropy of the $g$ factor of $\mathrm{Ti}_{\mathrm{B}}$. (a) ESR spectra of $\mathrm{Ti}_{\mathrm{v}}$ (upper) and $\mathrm{Ti}_{\mathrm{h}}$ (lower) at different angles $\theta$ of a vector magnetic field (same field magnitude) with tip $3\left(V_{\mathrm{DC}}=40 \mathrm{mV}, V_{\mathrm{RF}}=10 \mathrm{mV}, I_{\mathrm{set}}=20 \mathrm{pA}, T=1.12 \mathrm{~K}\right.$, and $\left.B_{\text {ext }}=0.7 \mathrm{~T}\right)$. (b) Current dependence of the resonance frequency of $\mathrm{Ti}_{\mathrm{B}}$ measured with tip 2. Solid lines are fits considering the exchange interaction between $\mathrm{STM}$ tip and $\mathrm{Ti}_{\mathrm{B}}$. We give error bars with $95 \%$ confidence interval $\left(V_{\mathrm{DC}}=40 \mathrm{mV}, V_{\mathrm{RF}}=10 \mathrm{mV}, T=1.12 \mathrm{~K}\right.$, and $\left.B_{\mathrm{ext}}=0.55 \mathrm{~T}\right)$. (c) $f_{0}$ as a function of the angle $\theta$. Each curve is fit to an ellipse in the $x z$ plane, tracing along the shape of the two-dimensional anisotropic $g$ factor. Error bars are from the fit in (b). (d) ESR frequency extrapolated to zero current $\left(f_{0}\right)$ at different magnitudes of $\boldsymbol{B}_{\text {ext }}$ along the out-of-plane (left) and in-plane (right) directions. We obtained the $g$ factors of $\mathrm{Ti}_{\mathrm{v}}$ and $\mathrm{Ti}_{\mathrm{h}}, g_{\mathrm{v} \|}=1.679 \pm 0.003$ and $g_{\mathrm{h} \|}=1.901 \pm 0.020$ at the in-plane field direction, and $g_{\mathrm{v} \perp}=1.975 \pm 0.011$ and $g_{\mathrm{h} \perp}=1.991 \pm 0.019$ at the out-of-plane field direction $\left(V_{\mathrm{DC}}=40 \mathrm{mV}, V_{\mathrm{RF}}=10 \mathrm{mV}\right.$, and $\left.T=1.12 \mathrm{~K}\right)$. We note that the fit has a subtle offset near zero, which might come from a non-negligible dipole-dipole coupling on the extrapolated $f_{0}$.

$g$ factor along all three spatial directions by a series of ESR measurements.

An example of a typical ESR spectrum for Ti at the bridge binding site is shown in Fig. 1(c), measured by sweeping the frequency $\left(f_{\mathrm{RF}}\right)$ of a radio-frequency voltage $\left(V_{\mathrm{RF}}\right)$, at a constant external magnetic field $\boldsymbol{B}_{\text {ext }}$. The ESR data were fitted to Eq. (1):

$$
I=I_{0}+\Delta I=I_{0}+I_{1} \frac{1+\alpha \delta}{1+\delta^{2}}
$$

where $I_{0}$ is the background current, $\Delta I$ is the ESR signal, $\delta$ is the normalized frequency $\left(\delta=\frac{f-f_{\text {res }}}{\Gamma / 2}\right)$ for the resonance frequency $f_{\text {res }}$ and the linewidth $\Gamma, I_{1}$ is the amplitude of the current change of the ESR signal, and $\alpha$ is the asymmetry factor [18]. When $\alpha$ is zero, Eq. (1) corresponds to the Lorentzian function. From the fit shown in Fig. 1(c), we obtained a resonance frequency $f_{\text {res }}=18.361 \pm 0.001 \mathrm{GHz}$ and the amplitude of the spectrum $I_{1}=835 \pm 11 \mathrm{fA}$ at $B_{\text {ext }}=0.7 \mathrm{~T}$ and tunnel current $I_{\text {set }}=20 \mathrm{pA}$.

\section{B. Mapping the $\boldsymbol{g}$-factor anisotropy of Ti atoms}

To obtain a full three-dimensional map of the magnetic environment, we measured ESR spectra on Ti at two inequivalent bridge binding sites. Note that we define $\theta$ as the angle between the surface normal and the magnetic field direction. We vary the angle $\theta$ as well as the magnitude of the external magnetic field $\left(B_{\text {ext }}\right)$ in a two-dimensional plane. Interestingly, we found a pronounced difference in the ESR spectra depending on the angle $\theta$ when measuring on $\mathrm{Ti}_{\mathrm{v}}$ and $\mathrm{Ti}_{\mathrm{h}}$ [Fig. 2(a)], while the resonance frequencies of both $\mathrm{Ti}_{\mathrm{v}}$ and $\mathrm{Ti}_{\mathrm{h}}$ increased and became more similar as we applied the magnetic field closer to the out-of-plane direction, indicating strong in-plane anisotropy. The observed shift of the ESR frequency of $\mathrm{Ti}_{\mathrm{v}}$ with the angle $\theta$ of the magnetic field is almost three times larger than that of $\mathrm{Ti}_{\mathrm{h}}$ [Fig. 2(a)].

The change of ESR frequency at different angles of $\boldsymbol{B}_{\text {ext }}$ results from the $g$-factor anisotropy of $\mathrm{Ti}$ atoms on $\mathrm{MgO}$, which has also been found for Ti absorbed on the oxygen binding site [29]. In order to precisely determine the $\boldsymbol{B}_{\text {ext }}$ angle 

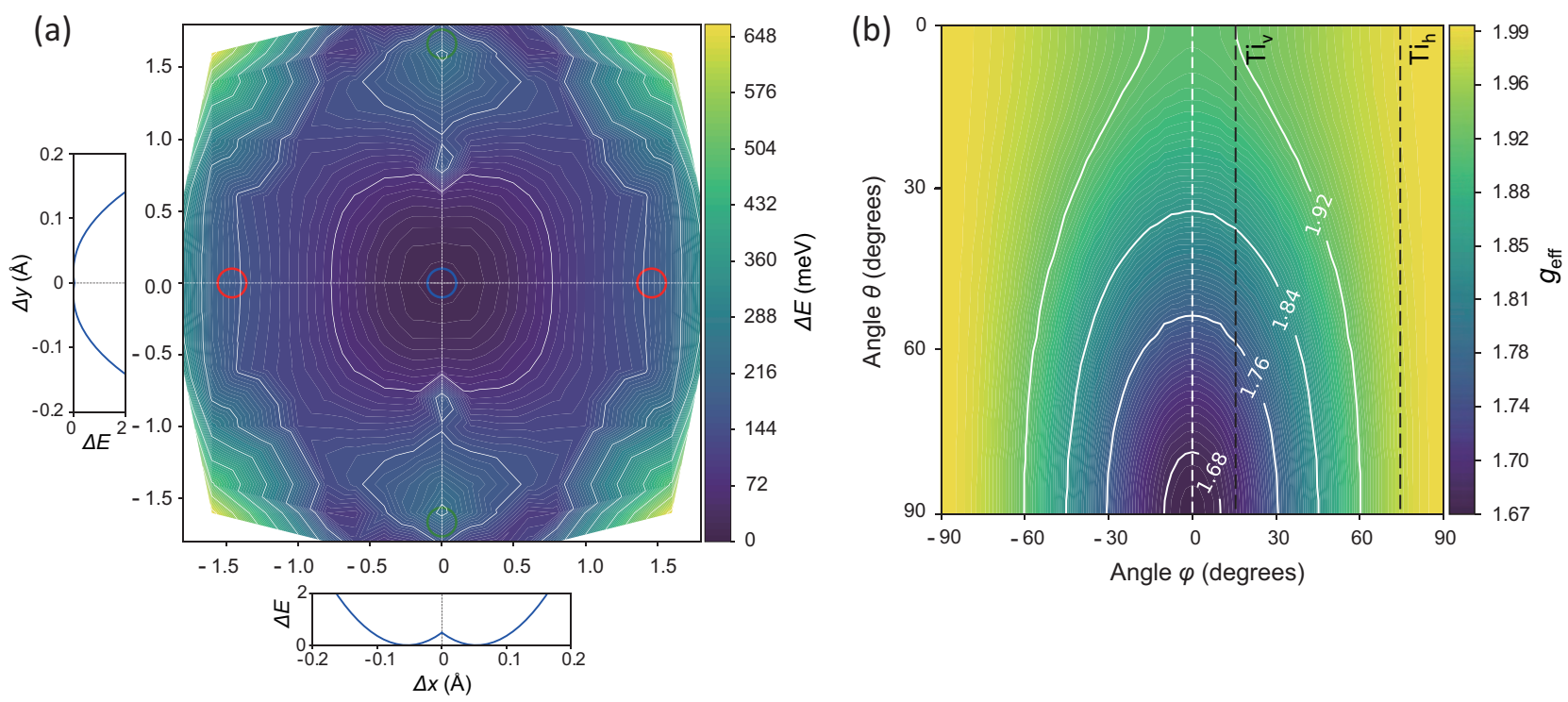

FIG. 3. Three-dimensional map of anisotropic $g$ factor and dynamic instability of a hydrogen atom on Ti. (a) Potential energy surface (PES) of a hydrogen atom on $\mathrm{Ti} / \mathrm{MgO}$. The Ti atom is located in the center of the figure with the $x$ axis pointing towards $\mathrm{O}$ located at the red circles and the $y$ axis towards $\mathrm{Mg}$ atoms (green). The insets show a line cut along $x$ and $y$ axis close to the center, clearly showing a pronounced double-well potential along the $x$ direction. (b) Effective $g$ factor plotted as functions of the in-plane angle $(\varphi)$ and out-of-plane angle $(\theta)$ of $\boldsymbol{B}_{\text {ext }}$ as obtained from the multiplet calculations. The same values of the $g$ factor are represented by contour lines. The values corresponding to $\mathrm{Ti}_{\mathrm{v}}$ and $\mathrm{Ti}_{\mathrm{h}}$ are indicated by vertical dashed lines, considering the symmetry of the MgO lattice.

dependence of the resonance frequency, it is necessary to eliminate the influence of the effective magnetic field created by a magnetic tip $\left(\boldsymbol{B}_{\text {tip }}\right)$ by measuring the ESR spectra on Ti spin at different tip-Ti distances [30]. At a given $\boldsymbol{B}_{\text {ext }}$, the ESR frequency shifts with tip-atom separation $d$ set by the tunnel current [Fig. 2(b)]. This shift is governed by two distinct terms of the tip field [16,31], the exchange field $B_{\text {exc }} \propto \exp \left(-d / d_{\text {ex }}\right)$ and the dipolar field $B_{\text {dip }} \propto 1 / d^{3}$. We found that the exchange field dominates for the tips used here, in line with several previous studies $[18,32,33]$. Extrapolating the resonance frequency $f_{0}$ at zero tunnel current allows us to determine the Zeeman energy depending only on the external magnetic field $\boldsymbol{B}_{\text {ext }}$.

The extrapolated resonance frequency $f_{0}$ is plotted as a function of the field angle $\theta$ in Fig. 2(c) at a constant magnitude of $B_{\text {ext }}=0.55 \mathrm{~T}$. Near the out-of-plane direction $(\theta=$ $\left.0^{\circ}\right), \mathrm{Ti}_{\mathrm{v}}$ and $\mathrm{Ti}_{\mathrm{h}}$ show the same ESR frequency. The resonance frequencies for $\mathrm{Ti}_{\mathrm{v}}$ and $\mathrm{Ti}_{\mathrm{h}}$ start deviating when rotating the field to the in-plane direction and eventually differ by about $1.54 \mathrm{GHz}$ at the in-plane magnetic field $\left(\theta=90^{\circ}\right)$. Unlike Ti at an oxygen binding site on the same $\mathrm{MgO}$ surface [29], the different in-plane resonance frequencies of $\mathrm{Ti}_{\mathrm{v}}$ and $\mathrm{Ti}_{\mathrm{h}}$ reflect the lower twofold symmetry of the bridge binding site.

To determine the $g$ factor along the three principal axes, we measured $f_{0}$ for both $\mathrm{Ti}_{\mathrm{v}}$ and $\mathrm{Ti}_{\mathrm{h}}$ as a function of magnetic field magnitudes $B_{\mathrm{ext}}$ at the magnetic field angles $\theta=0^{\circ}$ and $90^{\circ}$ [Fig. 2(d)]. From these measurements, the magnetic moments of the two $\mathrm{Ti}$ atoms were obtained from the linear fit of $f_{0}$ as a function of $B_{\text {ext }}$, which yields in-plane magnetic moments $\mu_{\mathrm{v} \|}=(0.840 \pm 0.001) \mu_{\mathrm{B}}$ and $\mu_{\mathrm{h} \|}=(0.950 \pm 0.010) \mu_{\mathrm{B}}$ for $\mathrm{Ti}_{\mathrm{v}}$ and $\mathrm{Ti}_{\mathrm{h}}$, respectively. The out-of-plane magnetic moments for $\mathrm{Ti}_{\mathrm{v}}$ and $\mathrm{Ti}_{\mathrm{h}}$ are $\mu_{\mathrm{v} \perp}=$ $(0.987 \pm 0.006) \mu_{\mathrm{B}}$ and $\mu_{\mathrm{h} \perp}=(0.996 \pm 0.010) \mu_{\mathrm{B}}$. Given that in our sample the $\mathrm{O}-\mathrm{O}$ bond direction (along the nearest neighbor oxygen atoms) of the $\mathrm{MgO}$ lattices was misaligned by $15.5^{\circ}$ with the in-plane component of the external magnetic field [Fig. 1(b)], we were able to determine the $g$ factors along the lattice directions: $g_{\mathrm{O}-\mathrm{O}}=1.653 \pm 0.002, g_{\mathrm{Mg}-\mathrm{Mg}}=$ $1.917 \pm 0.019$, and $g_{\mathrm{z}}=1.989 \pm 0.024$. We confirmed that the result of an anisotropic $g$ factor is reliable with several different tips and on other Ti atoms (see Sec. S6 in the Supplemental Material [17]).

\section{Theoretical analysis of $\boldsymbol{g}$-factor anisotropy}

To unravel the origin of the $g$-factor anisotropy along different axes of the MgO lattice, we calculated the effective $g$ factor along arbitrary directions of the external magnetic field $\boldsymbol{B}_{\text {ext }}$ [Fig. 3(b)] using a combination of DFT and magnetic multiplet calculations [34]. The hydrogenated $\mathrm{Ti}$ atoms in this system are known to have a dynamical instability of the hydrogen as the potential energy surface (PES) exhibits a double-well potential profile along the $x$ axis, with minima pointing towards the oxygen atoms (see also Sec. S5 in the Supplemental Material [17]). We accounted for this instability in the multiplet calculations by sampling the PES of hydrogen in the presence of $\mathrm{Ti}$ and the $\mathrm{MgO}$ substrate [Fig. 3(a)]. We performed multiplet calculations to obtain the $g$ factor under small displacements ( $\Delta x, \Delta y$; see Table I) of the hydrogen atom relative to the Ti. The resulting $g$ factor for each calculation was then weighted according to its statistical Boltzmann weight at $T=1.1 \mathrm{~K}$ with the energy obtained from the PES calculation $[E=f(\Delta x, \Delta y, \Delta z)]$. The calculated values for the $g$ factor along the principal axes are in excellent agreement with the experiment as shown in Table II. 
TABLE I. Point charge model used for the crystal field in the multiplet calculation. Shown are the (Lowdin) charges $(-|e|)$ and Cartesian coordinates for each ion in the crystal field along the three different spatial directions, where $Z$ is the out-of-plane direction and $\mathrm{O}-\mathrm{O}$ and $\mathrm{Mg}-\mathrm{Mg}$ represent the directions connecting each atom in the $\mathrm{MgO}$ lattice. All coordinates are relative to the Ti adatom.

\begin{tabular}{lcccc}
\hline \hline Ion & $\Delta$ electrons (no.) & $\mathrm{O}-\mathrm{O}(\AA)$ & $\mathrm{Mg}-\mathrm{Mg}(\AA)$ & $\mathrm{Z}(\AA)$ \\
\hline $\mathrm{O}$ & +0.75 & -1.36 & 0.0 & -1.27 \\
$\mathrm{O}$ & +0.75 & 1.38 & 0.0 & -1.27 \\
$\mathrm{Mg}$ & -0.73 & 0.0 & -1.67 & -2.10 \\
$\mathrm{Mg}$ & -0.73 & 0.0 & 1.67 & -2.10 \\
$\mathrm{H}$ & +0.46 & $0.00 \pm \Delta x$ & $0.00 \pm \Delta y$ & $1.76 \pm \Delta z$ \\
\hline \hline
\end{tabular}

The multiplet calculations also reveal the origin of the anisotropy to lie in a gradual reduction of the orbital moment which is coupled antiparallel to the spin when rotating the external magnetic field direction from the in-plane to the outof-plane direction. The magnitude of the reduction depends on the azimuthal angle as shown in Fig. 4, which indicates the contribution of the orbital moment is less than about $0.1 \mu_{\mathrm{B}}$ in the plane $\left(\theta=90^{\circ}\right)$. This presence of orbital momentum strongly affects the total momentum through the spin-orbit coupling, causing the anisotropy of the $g$ factor.

\section{ESR amplitude analysis and the model fit}

We will now focus on the change of amplitudes of ESR signals when rotating the external magnetic field. Knowing the intrinsic properties of the $\mathrm{Ti}$ spin on $\mathrm{MgO}$, we can use it as a probe to investigate how the magnetic interaction with the tip influences the ESR signals, which might hint why the spin polarization of the tip does not guarantee measurement of ESR signals. Note that measured Ti atoms are well isolated from other atoms on the surface (Supplemental Material [17]) but the tip spin is positioned on top. Two examples of ESR spectra for Ti measured with different tips as a function of the magnetic field direction $\theta$ are given in Figs. 2(a) and 5(a), which show rather dramatic changes of the amplitude of the ESR signals as a function of $\theta$. This variation is a consequence of the different relative alignment between the Ti spin and the unknown spin of the magnetic cluster at the tip. Considering the piezoelectric coupling (PEC) model for a single atom ESR in STM, the spin at the tip plays two important roles: (i) providing an oscillating magnetic field to drive the ESR transitions $[33,35]$ and (ii) detecting the ESR signals based on the tunneling magnetoresistance effect $[18,36]$.

In the PEC model, the Ti atoms experience a time-varying magnetic field under the spatially inhomogeneous effective

TABLE II. Values for the $g$ factor obtained from experiment and multiplet calculations.

\begin{tabular}{lcc}
\hline \hline$g$-factor element & Experiment & Multiplet \\
\hline$g_{\mathrm{O}-\mathrm{O}}$ & $1.653 \pm 0.002$ & 1.67 \\
$g_{\mathrm{Mg}-\mathrm{Mg}}$ & $1.917 \pm 0.019$ & 1.91 \\
$g_{\mathrm{z}}$ & $1.989 \pm 0.024$ & 1.99 \\
\hline \hline
\end{tabular}
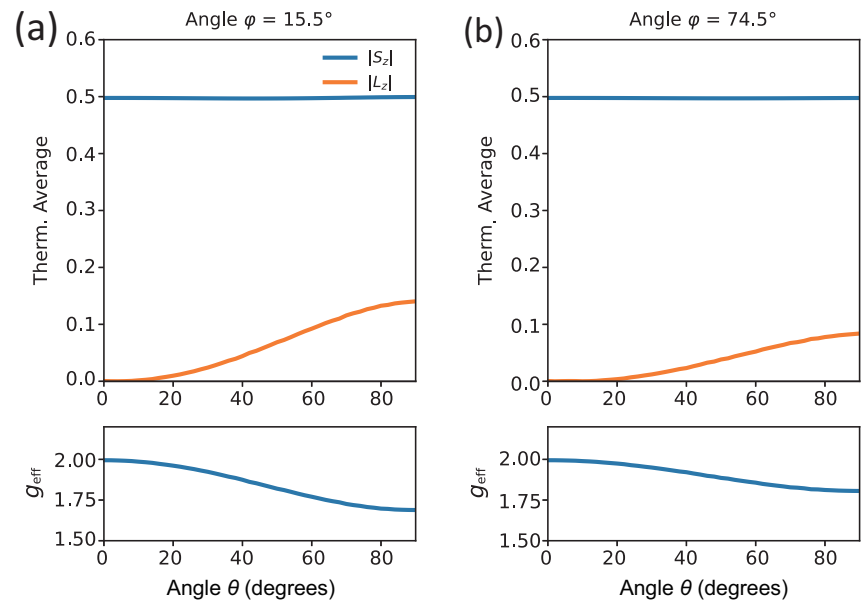

FIG. 4. Thermal averages at $1.1 \mathrm{~K}$ for the expectation value of spin and orbital moment along the external magnetic field direction for two azimuthal values $\varphi=15.5^{\circ}\left(\mathrm{Ti}_{\mathrm{v}}\right)$ (a) and $\varphi=74.5^{\circ}\left(\mathrm{Ti}_{\mathrm{h}}\right)$ (b) for changing $\theta$ measured from the out-of-plane direction. The anisotropy of the $g$ factor is the result of the antiparallel coupling of orbital and spin moment.

magnetic field, $\boldsymbol{B}_{\text {tip }}$, as a consequence of the adiabatic coupling of the motion of the Ti to the rf voltage $[32,35]$. To explain our data, we assume that the tip spin has a strong easyaxis magnetic anisotropy, which keeps the tip spin aligned in a certain direction fixed in the laboratory frame while $\boldsymbol{B}_{\text {ext }}$ changes. However, we have to consider that the tip spin can flip by $180^{\circ}$ to minimize its Zeeman energy (i.e., it is pointing up or down along its easy axis), which is indicated by the $180^{\circ}$ periodicity of the signal [Fig. 5(b)]. Figure 5(c) introduces the coordinates used in the model. The $x y z$ coordinates are determined by the surface normal ( $z)$ and the plane of $\boldsymbol{B}_{\text {ext }}(x z$ plane) as defined in Fig. 1(a), whereas the $x^{\prime} y^{\prime} z^{\prime}$ coordinate is defined to have the $z^{\prime}$ axis along the quantization axis of the Ti spin fixed by the direction of the external magnetic field. Note that we assume $\boldsymbol{B}_{\text {ext }}+\boldsymbol{B}_{\text {tip }} \approx \boldsymbol{B}_{\text {ext }}$ for ESR amplitude analysis.

The simplified equation for the ESR amplitude $I_{1}$ based on the PEC model is given by

$$
I_{1} \propto \Phi(\Omega)\left[\left\langle\boldsymbol{S}_{\mathrm{tip}}\right\rangle \cdot\left\langle\boldsymbol{S}_{\mathrm{Ti}}\right\rangle\right],
$$

where $\Omega$ is the Rabi rate and $\Phi(\Omega)=\Omega^{2} T_{1} T_{2} /\left(1+\Omega^{2} T_{1} T_{2}\right)$ is the driving factor on resonance, which ranges from 0 to 1 depending on how far the spin population is driven into equal state population [37,38]. Equation (2) shows that the ESR signals are determined by the product of the driving strength $(\Phi(\Omega))$ and the spin contrast for the detection $\left(\left\langle\boldsymbol{S}_{\mathrm{tip}}\right\rangle \cdot\left\langle\boldsymbol{S}_{\mathrm{Ti}}\right\rangle\right)$. The detailed fit function is described in Secs. S7 and S8 in the Supplemental Material [17].

As hinted in Eq. (2), rotating the magnetic field impacts driving and detection simultaneously. The influence on driving the ESR transition can be understood by assuming the quantization axis of the Ti spin $\boldsymbol{S}_{\mathrm{Ti}}$ along the $\boldsymbol{z}^{\prime}$ direction; the $x^{\prime}$ and $y^{\prime}$ components of $\boldsymbol{B}_{\text {tip }}$ provide the driving magnetic field $\boldsymbol{B}_{1}$ of ESR transitions, where we choose $\boldsymbol{y}^{\prime}=\boldsymbol{y}$. Since the Ti spin is aligned along the external magnetic field, rotating this field in the $x z$ plane results in a corresponding exchange of the amplitudes of the $x^{\prime}$ and $z^{\prime}$ components of $\boldsymbol{B}_{\text {tip }}$ as 


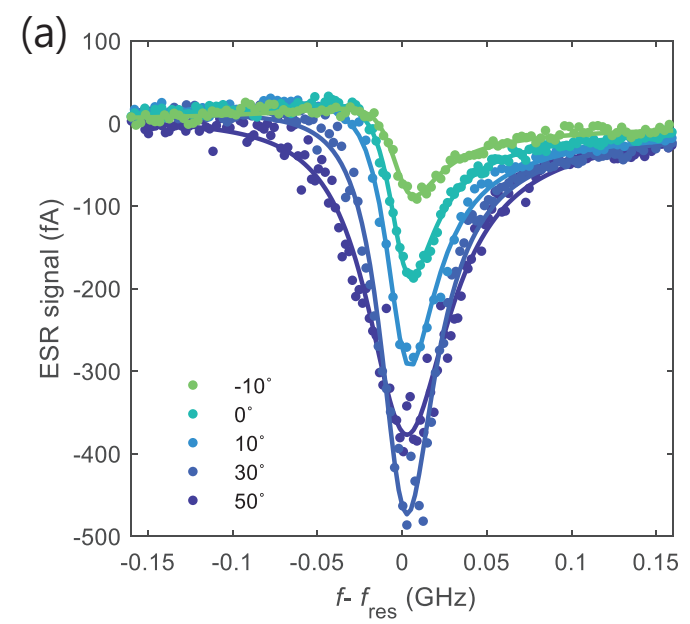

(c)

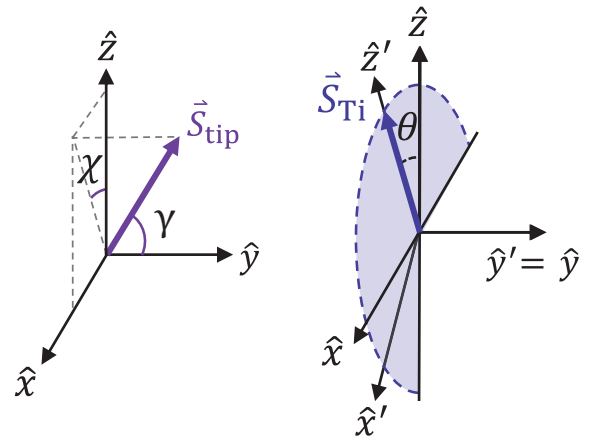

(b)

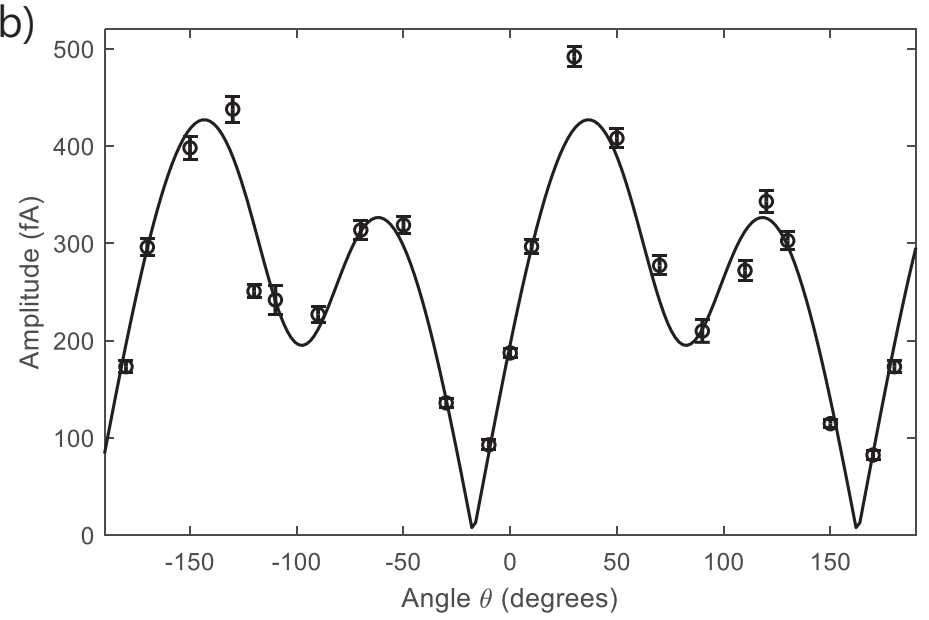

(d)

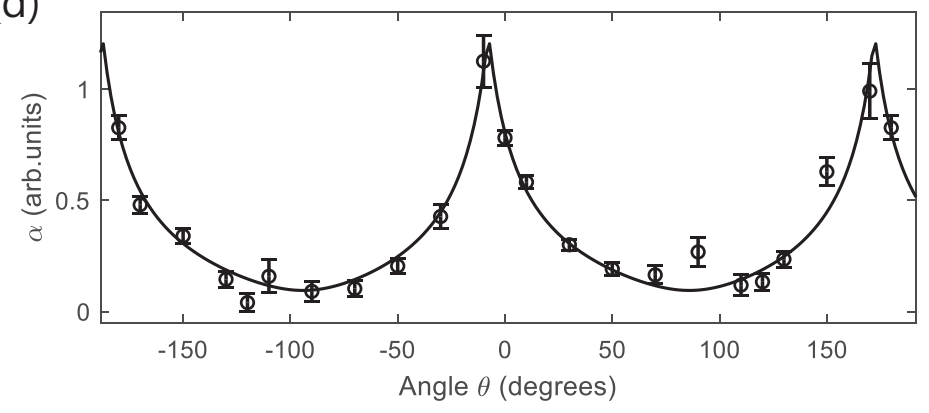

FIG. 5. ESR amplitudes at different directions of magnetic fields for $\mathrm{Ti}_{\mathrm{h}}$ as a probe of the tip magnetic field. (a) ESR spectra at different angles $(\theta)$ of the external magnetic field using tip $1\left(V_{\mathrm{DC}}=40 \mathrm{mV}, I=20 \mathrm{pA}, V_{\mathrm{RF}}=10 \mathrm{mV}, T=1.12 \mathrm{~K}\right.$, and $\left.B_{\text {ext }}=0.7 \mathrm{~T}\right)$. (b) ESR amplitude as a function of the magnetic field angle, $\theta$. The ESR amplitude of each point is extracted from the fit of ESR curves in (a) to Eq. (1). The solid line is a fit to the model described in the main text and Secs. S7 and S8 in the Supplemental Material [17]. (c) Schematic of the relative spin orientations of tip (left) and sample (right). We define the $x$ and $z$ axis as in-plane and out-of-plane directions of the sample surface, respectively, as defined in Fig. 1(a). The direction of the tip spin is denoted by the angles $\gamma$ and $\chi$ with respect to the $y$ and $z$ axes in the laboratory frame. At the right, the rotated coordinate system, where the $z^{\prime}$ axis is the quantization axis of the Ti spin, is given to have the $x^{\prime} z^{\prime}$ plane lie on the $x z$ plane in the laboratory frame since we rotate $\boldsymbol{B}_{\text {ext }}$ in that plane $\left(y^{\prime}=y\right)$. (d) Asymmetry factor $\alpha$ as a function of $\theta$. The asymmetry factor $\alpha$ is extracted from the same fit used in (b). It describes the relative contribution of homodyne detection over the DC magnetoconductance, depending on the alignment of the tip spin and Ti spin.

experienced by the Ti spin. The strength of $\boldsymbol{B}_{1}$ consequently changes depending on the amplitude of the $x^{\prime}$ component of $\boldsymbol{B}_{\text {tip }}$, while the $y^{\prime}$ component of $\boldsymbol{B}_{\text {tip }}$ provides a constant $\boldsymbol{B}_{1}$ field (independent of the angle $\theta$ ). Since $\Omega$ is proportional to the amplitude of the driving magnetic field, the driving factor in Eq. (2) varies accordingly [see the dashed line in Fig. 6(d)].

The detection of ESR signals in STM is based on the tunneling magnetoresistance effect. In STM, we measure the time-averaged tunneling current [14], which is detected by the applied DC and rf bias voltages [18]. The ESR signal detected by $V_{\mathrm{DC}}$ corresponds to the change in the time-averaged population of spin states detected by the component of the tip spin that is parallel to the quantization axis (the $z^{\prime}$ component of $\left.\boldsymbol{S}_{\text {tip }}\right)$. The AC contribution to the ESR signal detected by $V_{\mathrm{RF}}$ results from the rectification of the applied $V_{\mathrm{RF}}$ by the spin precession at the same frequency $[39,40]$. This oscillating conductance is detected by the $x^{\prime}$ and $y^{\prime}$ components of $\boldsymbol{S}_{\text {tip }}$. The ESR signal corresponding to the DC magnetoconductance shows four peaks when $\theta$ varies by $360^{\circ}$, since the detection is maximum when $\boldsymbol{S}_{\text {tip }}$ is parallel to the quantization axis of the Ti atom $\left(\boldsymbol{S}_{\mathrm{Ti}}\right)$ and the driving is maximum when
$\boldsymbol{B}_{\text {tip }}$ is perpendicular to $\boldsymbol{S}_{\mathrm{Ti}}$. The AC magnetoconductance component (or homodyne detection) shows only two maxima in the ESR amplitude since both driving and detection are maximum when $\boldsymbol{S}_{\text {tip }}$ is perpendicular to $\boldsymbol{S}_{\mathrm{Ti}}$. The total ESR signal (AC and DC contributions) then results in the four maxima observed in Fig. 5(b).

Interestingly, when we assume that $\boldsymbol{B}_{\text {tip }}$ is exactly parallel with the spin polarization of the tip $\boldsymbol{S}_{\text {tip }}$, we are unable to produce different height of the peaks shown in Fig. 5(b) using Eq. (2) (see the details in Sec. S8 in the Supplemental Material [17]). Instead, having $\boldsymbol{B}_{\text {tip }}$ slightly different from the $\boldsymbol{S}_{\text {tip }}$ direction, $\boldsymbol{B}_{\text {tip }} \neq J(z)\left\langle\boldsymbol{S}_{\text {tip }}\right\rangle$, based on their different origins, yields the best fit as given by the solid line in Fig. 5(b). The difference can occur from the non-negligible dipole interaction between the tip and the atom. Furthermore, while the spin polarization $S_{\text {tip }}$ in the tunneling process is determined by the spin-resolved density of states at the Fermi level [36,41], the tip magnetization $\boldsymbol{B}_{\text {tip }}$ for the ESR driving fields results from the total occupied states of the tip spin. Such different origins describe well the results in Fig. 5(b) with different peak height. The rounded-out dips correspond to minima in 

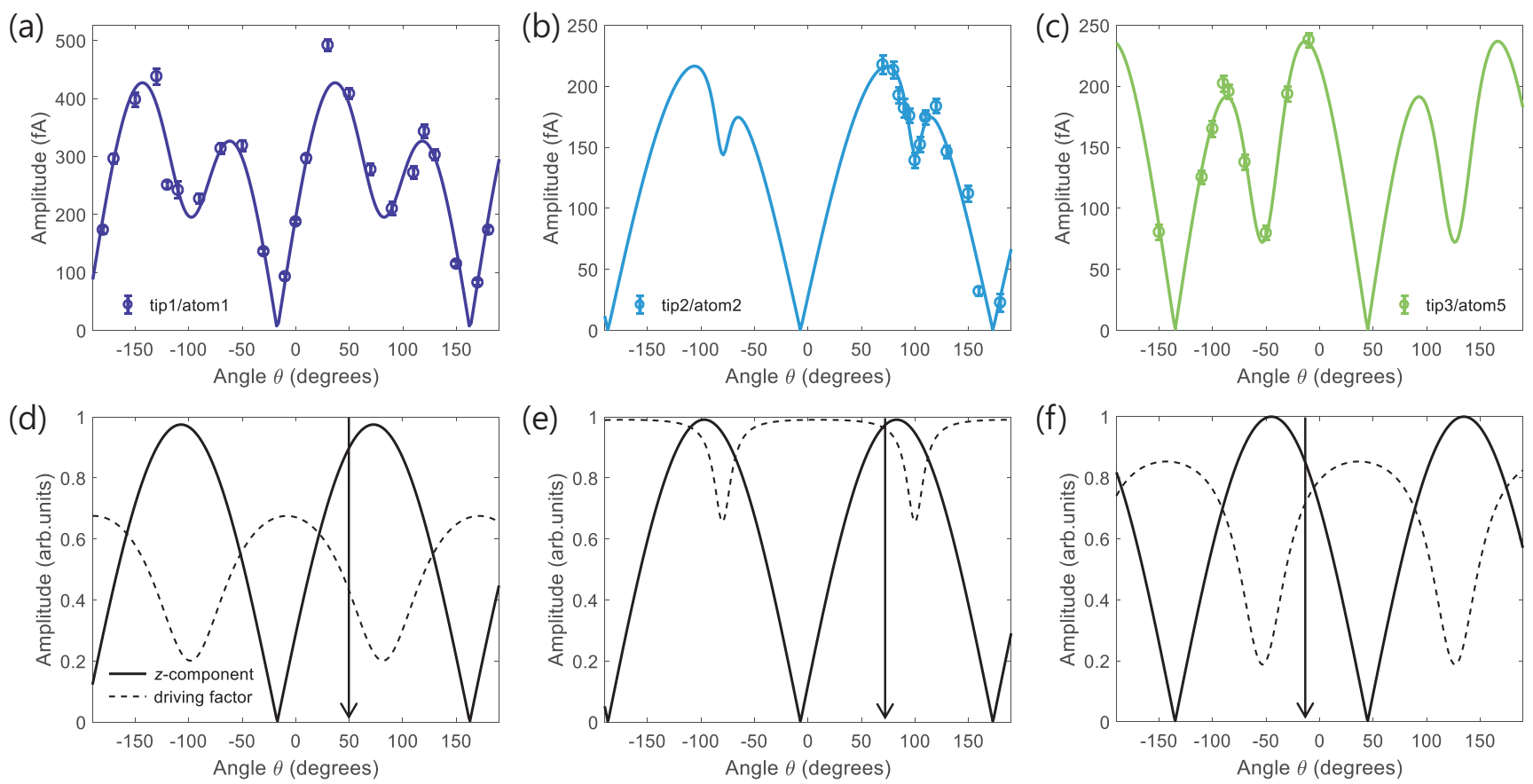

FIG. 6. ESR amplitude with model fits for three different tips: (a)-(c) model fits of ESR amplitude with data points obtained at $V_{\mathrm{DC}}=$ $40 \mathrm{mV}, I=20 \mathrm{pA}, V_{\mathrm{RF}}=10 \mathrm{mV}$, and $T=1.12 \mathrm{~K}$ and (d)-(f) contribution of the $z^{\prime}$ component of $\boldsymbol{S}_{\text {tip }}$ (DC magnetoconductance, solid line) and driving factor $\Phi(\Omega)$ (dashed line) to the fit for tips 1-3, respectively. The results for tip 2 were measured at $B_{\text {ext }}$ of 0.55 T and the results with the other two tips were measured at $0.7 \mathrm{~T}$. We made the spin-polarized tip for ESR measurement at $\boldsymbol{B}_{\text {ext }}$ with $\theta=50^{\circ}$ for tip $1,70^{\circ}$ for tip 2 , and $-10^{\circ}$ for tip 3 [marked by arrow lines in (d)-(f)]. At those angles, the ESR signal appears maximum as we prepared the tip to yield large ESR signal amplitude $I_{1}$.

the driving field, which corresponds to the minimum $\boldsymbol{B}_{1}$. On the other hand, the sharp dips in Fig. 5(b) appear at $\approx-18^{\circ}$ and $\approx 162^{\circ}$, where the DC magnetoconductance (depending on $\boldsymbol{S}_{\text {tip }}$ ) is minimum and $\boldsymbol{S}_{\text {tip }}$ flips by $180^{\circ}$.

The amount of homodyne signals over the DC detection can be recognized from the asymmetry factor in Fig. 5(d). The asymmetric line shape of ESR signals originates from the homodyne detection of the tunneling current [18,32]. The maximum of the asymmetry factor, thus, corresponds to the maximum of the $x^{\prime}$ and $y^{\prime}$ components of $S_{\text {tip }}$, which allows us to determine the direction of $S_{\text {tip }}$ in the laboratory frame.

\section{E. Contributions of the spin-polarized tip to the ESR amplitude}

We found a remarkable degree of reproducibility of the basic features of the $\boldsymbol{B}_{\text {ext }}$ field angle dependence of ESR signals for different tips and different Ti atoms. The ESR amplitudes as a function of the $\boldsymbol{B}_{\text {ext }}-$ field angle for three different tips are given in Figs. 6(a)-6(c).

We found that the ESR signals are almost at the maximum in amplitude at the angle at which we prepared the spin-polarized STM tip to measure ESR. This is due to the preparation procedure which aims to maximize the ESR signal at the preparation angle. As the total ESR amplitude is the result of the product between ESR driving and ESR detection [Eq. (2)], the strongest ESR signals are found at the angle where both driving strength and magnetoconductance are large. The ESR signal amplitude vanishes at certain angles, which explains why some spin-polarized tips do not give any ESR signals empirically. The contributions of the $z^{\prime}$ component of $\boldsymbol{S}_{\text {tip }}$ for the ESR detection and the $B_{\text {tip }}$ to the driving factor $\Phi(\Omega)$, as extracted from the fit, are depicted in Figs. 6(d)-6(f) by solid and dotted lines, respectively. While the driving factor is nonzero at every angle due to the finite $y^{\prime}$ components of $\boldsymbol{B}_{\text {tip }}$, independent of $\boldsymbol{B}_{\text {ext }}$ angle, the $z^{\prime}$ component of $\boldsymbol{S}_{\text {tip }}$ is zero at certain angles, at which no ESR signals are observed. Based on this simple model [Eq. (2) and Supplemental Material [17]], the amplitudes of the ESR signals for different tips are well described as shown by the fit curves in Fig. 6.

We further confirmed that the direction of $\boldsymbol{B}_{\text {tip }}$ and $\boldsymbol{S}_{\text {tip }}$ varies for different tips. From the fit, we were able to distinguish the direction of $\boldsymbol{S}_{\text {tip }}$ and $\boldsymbol{B}_{\text {tip }}$ in three dimensions, where both $\boldsymbol{S}_{\text {tip }}$ and $\boldsymbol{B}_{\text {tip }}$ are off from the rotating plane of the external magnetic fields. In the case of tip 2 [Figs. 6(b) and 6(e)], the difference between the direction of $\boldsymbol{B}_{\text {tip }}$ and $\boldsymbol{S}_{\text {tip }}$ is $\approx 17^{\circ}$, which is bigger than other tips (less than $\approx 10^{\circ}$ ) (see Sec. $S 8$ in the Supplemental Material [17]). This larger difference of $\boldsymbol{B}_{\text {tip }}$ and $\boldsymbol{S}_{\text {tip }}$ results in a less balanced distribution of the amplitude of the peaks. The detailed fitting parameters for three different tips are given in Sec. S8 in Supplemental Material [17].

This model demonstrates three critical points of the ESRSTM mechanism.

(1) The direction of the spin polarization and the magnetic field by the tip can have a small mismatch depending on tips. Here, we derived those directions in three dimensions from the amplitude fit (Sec. S8 in Supplemental Material [17]).

(2) The largest ESR signal is observed when the tip spin is aligned in a way to have adequate $z^{\prime}$ component of $\boldsymbol{S}_{\text {tip }}$ 
and $x^{\prime}$ components of $\boldsymbol{B}_{\text {tip }}$. This ensures that ESR driving and detection are both effective.

(3) The DC magnetoconductance contributes more strongly to the ESR amplitude than the AC magnetoconductance at $V_{\mathrm{RF}}=10 \mathrm{mV}$.

The analysis on the three-dimensional direction of $\boldsymbol{S}_{\text {tip }}$ and $\boldsymbol{B}_{\text {tip }}$ provides further understanding of the tip-induced magnetic environment.

\section{CONCLUSIONS}

In this paper, we utilized a vector magnetic field to obtain a three-dimensional mapping of the $g$-factor anisotropy of $\mathrm{Ti}$ on $\mathrm{MgO}$, and the results show a high degree of agreement with the multiplet calculation. The degree and directionality of this anisotropy highlight the sensitivity of the Ti spin to the substrate crystal field. Using this well-characterized surface spin through extensive measurements of the ESR frequency, amplitude, and line shape of a single Ti spin in vector mag- netic fields, we fully characterized the tip-Ti coupling and determined the tip spin direction. Our paper further underlines the versatility of ESR-STM in a vector magnetic field as a highly sensitive magnetic probe to study the interaction between spin centers and their solid-state environments as well as the magnetic structure of the tip.

\section{ACKNOWLEDGMENTS}

This work was supported by the Institute for Basic Science (Grant No. IBS-R027-D1). D.-J.C. acknowledges financial support from the Spanish MICINN (Project No. RTI2018097895-B-C44 and Excelencia Project No. EUR2020112116) and Eusko Jaurlaritza (Project No. PIBA_2020_1_0017). F.D. acknowledges financial support from Spanish MICINN (Grant No. PID2019-109539GB-C41), Basque Government (Grant No. IT986-16), and Canary Islands program Viera y Clavijo (Grant No. 2017/0000231). C.P.L. acknowledges financial support from the ONR.
[1] C. L. Degen, F. Reinhard, and P. Cappellaro, Rev. Mod. Phys. 89, 035002 (2017).

[2] G. Czap, P. J. Wagner, F. Xue, L. Gu, J. Li, J. Yao, R. Wu, and W. Ho, Science 364, 670 (2019).

[3] M. Ormaza, N. Bachellier, M. N. Faraggi, B. Verlhac, P. Abufager, P. Ohresser, L. Joly, M. Romeo, F. Scheurer, M.-L. Bocquet, N. Lorente, and L. Limot, Nano Lett. 17, 1877 (2017).

[4] J. R. Petta, A. C. Johnson, J. M. Taylor, E. A. Laird, A. Yacoby, M. D. Lukin, C. M. Marcus, M. P. Hanson, and A. C. Gossard, Science 309, 2180 (2005).

[5] A. Ajoy, U. Bissbort, M. D. Lukin, R. L. Walsworth, and P. Cappellaro, Phys. Rev. X 5, 011001 (2015).

[6] J. J. Pla, K. Y. Tan, J. P. Dehollain, W. H. Lim, J. J. L. Morton, F. A. Zwanenburg, D. N. Jamieson, A. S. Dzurak, and A. Morello, Nature (London) 496, 334 (2013).

[7] G. Széchenyi, L. Chirolli, and A. Pályi, 2D Materials 5, 035004 (2018).

[8] J. Pawłowski, New J. Phys. 21, 123029 (2019).

[9] S. Nadj-Perge, S. M. Frolov, E. P. A. M. Bakkers, and L. P. Kouwenhoven, Nature (London) 468, 1084 (2010).

[10] J. J. Bollinger, J. D. Prestage, W. M. Itano, and D. J. Wineland, Phys. Rev. Lett. 54, 1000 (1985).

[11] A. J. Heinrich, J. A. Gupta, C. P. Lutz, and D. M. Eigler, Science 306, 466 (2004).

[12] F. Delgado and J. Fernández-Rossier, Phys. Rev. Lett. 107, 076804 (2011).

[13] C. R. Ast, B. Jäck, J. Senkpiel, M. Eltschka, M. Etzkorn, J. Ankerhold, and K. Kern, Nat. Commun. 7, 13009 (2016).

[14] S. Baumann, W. Paul, T. Choi, C. P. Lutz, A. Ardavan, and A. J. Heinrich, Science 350, 417 (2015).

[15] T. Choi, W. Paul, S. Rolf-Pissarczyk, A. J. Macdonald, F. D. Natterer, K. Yang, P. Willke, C. P. Lutz, and A. J. Heinrich, Nat. Nanotechnol. 12, 420 (2017).

[16] K. Yang, Y. Bae, W. Paul, F. D. Natterer, P. Willke, J. L. Lado, A. Ferrón, T. Choi, J. Fernández-Rossier, A. J. Heinrich, and C. P. Lutz, Phys. Rev. Lett. 119, 227206 (2017).
[17] See Supplemental Material at http://link.aps.org/supplemental/ 10.1103/PhysRevB.104.174408 for further details, including Secs. S1-S10, Figs. S1-S14, Tables S1-S4, and Eqs. (S1)(S25).

[18] Y. Bae, K. Yang, P. Willke, T. Choi, A. J. Heinrich, and C. P. Lutz, Sci. Adv. 4, eaau4159 (2018).

[19] A. Ferrón, F. Delgado, and J. Fernández-Rossier, New J. Phys. 17, 033020 (2015).

[20] P. Giannozzi, O. Andreussi, T. Brumme, O. Bunau, M. Buongiorno Nardelli, M. Calandra, R. Car, C. Cavazzoni, D. Ceresoli, M. Cococcioni, N. Colonna, I. Carnimeo, A. Dal Corso, S. de Gironcoli, P. Delugas, R. A. DiStasio, A. Ferretti, A. Floris, G. Fratesi, G. Fugallo, R. Gebauer, U. Gerstmann, F. Giustino, T. Gorni, J. Jia, M. Kawamura, H. Y. Ko, A. Kokalj, E. Küçükbenli, M. Lazzeri, M. Marsili, N. Marzari, F. Mauri, N. L. Nguyen, H. V. Nguyen, A. Otero-de-la-Roza, L. Paulatto, S. Poncé, D. Rocca, R. Sabatini, B. Santra, M. Schlipf, A. P. Seitsonen, A. Smogunov, I. Timrov, T. Thonhauser, P. Umari, N. Vast, X. Wu, and S. Baroni, J. Phys. Condens. Matter 29, 465901 (2017).

[21] G. Prandini, A. Marrazzo, I. E. Castelli, N. Mounet, and N. Marzari, npj Computational Materials 4, 72 (2018).

[22] S. Grimme, J. Comput. Chem. 25, 1463 (2004).

[23] A. Ferrón, S. A. Rodríguez, S. S. Gómez, J. L. Lado, and J. Fernández-Rossier, Phys. Rev. Research 1, 033185 (2019).

[24] P. O. Löwdin, J. Chem. Phys. 18, 365 (1950).

[25] C. Herrmann, M. Reiher, and B. A. Hess, J. Chem. Phys. 122, 034102 (2005).

[26] A. Abragam and B. Bleaney, Electron Paramagnetic Resonance of Transition Ions (Oxford University, New York, 2012).

[27] P. Willke, Y. Bae, K. Yang, J. L. Lado, A. Ferrón, T. Choi, A. Ardavan, J. Fernández-Rossier, A. J. Heinrich, and C. P. Lutz, Science 362, 336 (2018).

[28] L. M. Veldman, L. Farinacci, R. Rejali, R. Broekhoven, J. Gobeil, D. Coffey, M. Ternes, and A. F. Otte, Science 372, 964 (2021). 
[29] M. Steinbrecher, W. M. J. van Weerdenburg, E. F. Walraven, N. P. E. van Mullekom, J. W. Gerritsen, F. D. Natterer, D. I. Badrtdinov, A. N. Rudenko, V. V. Mazurenko, M. I. Katsnelson, A. van der Avoird, G. C. Groenenboom, and A. A. Khajetoorians, Phys. Rev. B 103, 155405 (2021).

[30] S. Yan, D.-J. Choi, J. A. J. Burgess, S. Rolf-Pissarczyk, and S. Loth, Nat. Nanotechnol. 10, 40 (2015).

[31] P. Willke, K. Yang, Y. Bae, A. J. Heinrich, and C. P. Lutz, Nat. Phys. 15, 1005 (2019).

[32] T. S. Seifert, S. Kovarik, D. M. Juraschek, N. A. Spaldin, P. Gambardella, and S. Stepanow, Sci. Adv. 6, eabc5511 (2020).

[33] K. Yang, W. Paul, F. D. Natterer, J. L. Lado, Y. Bae, P. Willke, T. Choi, A. Ferrón, J. Fernández-Rossier, A. J. Heinrich, and C. P. Lutz, Phys. Rev. Lett. 122, 227203 (2019).

[34] C. Wolf, F. Delgado, J. Reina, and N. Lorente, J. Phys. Chem. A 124, 2318 (2020).
[35] J. L. Lado, A. Ferrón, and J. Fernández-Rossier, Phys. Rev. B 96, 205420 (2017).

[36] R. Wiesendanger, Rev. Mod. Phys. 81, 1495 (2009).

[37] P. Willke, A. Singha, X. Zhang, T. Esat, C. P. Lutz, A. J. Heinrich, and T. Choi, Nano Lett. 19, 8201 (2019).

[38] P. Willke, W. Paul, F. D. Natterer, K. Yang, Y. Bae, T. Choi, J. Fernández-Rossier, A. J. Heinrich, and C. P. Lutz, Sci. Adv. 4, eaaq1543 (2018).

[39] H. Kubota, A. Fukushima, K. Yakushiji, T. Nagahama, S. Yuasa, K. Ando, H. Maehara, Y. Nagamine, K. Tsunekawa, D. D. Djayaprawira, N. Watanabe, and Y. Suzuki, Nat. Phys. 4, 37 (2008).

[40] T. Nozaki, Y. Shiota, S. Miwa, S. Murakami, F. Bonell, S. Ishibashi, H. Kubota, K. Yakushiji, T. Saruya, A. Fukushima, S. Yuasa, T. Shinjo, and Y. Suzuki, Nat. Phys. 8, 491 (2012).

[41] S. Loth, C. P. Lutz, and A. J. Heinrich, New J. Phys. 12, 125021 (2010). 